\title{
A Potential Predictive Biomarker for Miller/Payne Grading: PD-L1 Expression before Neoadjuvant Chemotherapy in Breast Cancer
}

\author{
Cheng Li ${ }^{\mathrm{a}}$ Rui-Zhong Mab Gui-Yan Hanc Ying-Hua Guo ${ }^{\mathrm{b}}$ Ya-Nan Zhang ${ }^{\mathrm{a}}$ \\ Ya-Ting Zhang ${ }^{a}$ Hui Wang ${ }^{c}$ Yu-Ping Zhang ${ }^{c}$ Fang-Ming Chen ${ }^{d}$ \\ Shi-Geng Zhang ${ }^{\mathrm{e}}$ Ming-Chen Wang ${ }^{\mathrm{b}}$ Fu-Rong Hao ${ }^{\mathrm{b}, \mathrm{f}}$ Yun-Xiang Zhang ${ }^{\mathrm{c}}$ \\ ${ }^{a}$ Clinical School, Weifang Medical University, Weifang, China; ${ }^{\mathrm{b}}$ Department of Radiation Oncology, Weifang People's \\ Hospital, Weifang, China; ${ }^{c}$ Department of Pathology, Weifang People's Hospital, Weifang, China; ${ }^{d}$ Department \\ of Radiation Oncology, Rongcheng People's Hospital, Weihai, China; ' ${ }^{\circ}$ Department of Radiation Oncology, Taian \\ Tumor Prevention and Treatment Hospital, Taian, China; 'Weifang Key Laboratory of Radiophysics and Oncological \\ Radiobiology, Weifang, China
}

\section{Keywords}

Breast cancer · Neoadjuvant chemotherapy · Immunohistochemical analysis - Tissue microarrays . Programmed death ligand $1 \cdot$ Miller/Payne system . Predictive biomarker

\begin{abstract}
Background and Objective: The aim of this study was to investigate the value of programmed death ligand 1 (PD-L1) expression as a predictive biomarker for Miller/Payne grading before neoadjuvant chemotherapy (NACT) in breast cancer. Patients and Methods: The expression of PD-L1 in pretreatment biopsies of breast cancer was assessed by immunohistochemistry in tissue microarrays. The results were analyzed using SPSS 22.0 statistical software. Results: Of 53 female patients, 10 (18.9\%) patients had a grade 5 (G5) response, and $12(22.6 \%)$ patients showed PD-L1 expression, including $7(13.2 \%)$ patients with staining in tumor cells (TCs) and 8 (15.1\%) patients with staining in peritumoral lymphocytes (PTLCs). Logistic regression analysis revealed that G5 response to NACT was significantly associated with TCs or PTLCS PD-L1 positivity, whether with univariate analysis (TCs PD-L1: $p=0.00$, OR 20.50, 95\% Cl 3.11-134.94; PTLCs PD-L1: $p=0.02$, OR 6.50, 95\% Cl 1.27-33.20) or with multivariate analysis (TCs PD-L1: $p=0.00, \mathrm{OR} 42.23,95 \% \mathrm{Cl}$ 3.36-530.90; PTLCs PD-L1: $p=0.02$, OR 9.07, 95\% Cl 1.37-
\end{abstract}


Table 1. Neoadjuvant chemotherapy regimens of 53 patients with breast cancer

\begin{tabular}{llc}
\hline NACT & Median cycle & Cases, $n(\%)$ \\
\hline CE & $4(2-4)$ & $6(11.3)$ \\
CE $\rightarrow$ T & $8(6-8)$ & $36(67.9)$ \\
TE & $6(6)$ & $4(7.5)$ \\
TEC & $6(6)$ & $6(11.3)$ \\
TB & $4(4)$ & $1(1.9)$ \\
\hline
\end{tabular}
el.

B, carboplatin; C, cyclophosphamide; E, epirubicin; T, docetax-

are not suitable for breast-conserving surgery [2, 3]. Recent research and clinical trials have shown a significant correlation of breast cancer responses to NACT with survival and prognosis $[4,5]$. Patients who achieve a pathologic complete response ( $\mathrm{pCR}$ ) to NACT tend to have improved disease-free survival (DFS) and overall survival (OS) compared with patients with residual invasive disease $[4,6-8]$. In a multivariate analysis of known prognostic factors, the Miller/Payne (MP) grading system was an independent predictor of OS or DFS [9]. Therefore, effectively predicting which patients can achieve grade 5 response/pCR before NACT is of great clinical significance.

At present, programmed death ligand 1 (PD-L1) and tumor-infiltrating lymphocytes (TILs) in the tumor microenvironment have become a research focus in many tumors and suggest immune evasion or immune resistance to endogenous antitumor activity; the positive expression of PD-L1 is also a common event in breast cancer [10-15]. However, the expression of PD-L1 in breast cancer cells or peritumoral lymphocytes (PTLCs) in pretreatment biopsies and its correlation with the response to NACT has not been extensively investigated. Here, we aim to investigate the correlation of MP grading with $\mathrm{PD}$ L1 expression before NACT and explore the predictive biomarker value of PD-L1 in pretreatment biopsies in the response to NACT in breast cancer.

\section{Materials and Methods}

\section{Patients and Clinicopathological Characteristics}

Female patients with primary breast cancer treated with NACT from January 1, 2016, to October 31, 2018, at Weifang People's Hospital were analyzed. The institution ethics review board approved this study. The criteria for inclusion were as follows: (1) histologically confirmed invasive carcinoma at core needle biopsy in our hospital; (2) both NACT and surgical procedures were performed in our hospital; (3) the MP grade was available after surgery; and (4) never received chemotherapy, radiation, endocrine therapy, or targeted therapies before NACT. A total of 75 patients were enrolled. The exclusion criteria were as follows: (1) the status of estrogen receptor (ER), progesterone receptor (PR), and HER2 expression and the Ki-67 score were not completely available for biopsy diagnoses; (2) breast cancer recurred for a second time after being treated; and (3) the biopsy sample is insufficient or severe artifacts present. Thus, 21 patients were excluded from the cohort only due to the last exclusion criterion. In addition, 1 case was excluded from the cohort due to deficient staining in the tissue. Finally, a total of 53 patients were included in the analysis of the results. The median age was 51.4 years (range 26.8-69.4).

\section{Treatment Methods}

All patients received 2-8 cycles of NACT, and the median cycle of NACT was 8 (Table 1). A total of 32.1\% (17/53) had HER2 expression, and $22.6 \%(12 / 53)$ received HER2 targeted therapies in addition to NACT.

Reagents

PD-L1 (CD274) Mouse Monoclonal Antibody (Clone ID: UMAB228) from OriGene Technologies (Beijing, China).

\section{Tissue Microarrays and Immunohistochemistry}

Briefly, representative tumor areas were selected in hematoxylin/eosin-stained slides, which served as a useful guide for selecting the area to be sampled, and 3-mm cores were obtained using a needle and seated into a recipient paraffin block with a spacing of $2 \mathrm{~mm}$ between the tissue microarrays (TMAs) samples. Once the TMAs were constructed, the samples were sectioned into $5-\mu \mathrm{m}$ continuous slides. The cases were evaluated on 3 previously constructed TMAs containing formalin-fixed, paraffin-embedded tissues from 53 invasive primary breast carcinomas. Next, the TMAs were stained using an immunohistochemistry (IHC) staining system, the Dako Autostainer Link 48 (NYSE:A, Palo Alto, CA, USA), according to their manual.

Serial pretreatment biopsies sections $(5 \mu \mathrm{m})$ were cut from all TMAs, mounted on slides, and baked for $1 \mathrm{~h}$ at $65^{\circ} \mathrm{C}$. The slides were de-paraffinized and rehydrated as follows: xylene I and II, 15 min, respectively; $100 \%$ ethanol, $3 \mathrm{~min}$; $95 \%$ ethanol, $3 \mathrm{~min} ; 75 \%$ ethanol, $3 \mathrm{~min}$; distilled water, $3 \mathrm{~min}$. The sections were antigenretrieved at $95^{\circ} \mathrm{C}$ for $20 \mathrm{~min}$ in the PT Link. This was followed by incubation with primary antibody for $30 \mathrm{~min}$, secondary antibody for $20 \mathrm{~min}$, and stained in diaminobenzidine. The slides were counterstained for 1-2 min with hematoxylin, differentiated for 10 $s$ with $0.1 \% \mathrm{HCl}$, washed with running water, blued for $30 \mathrm{~s}$ with ammonia water, dehydrated for 2 min using gradient ethanol washes $(75,95 \%$ I, 95\% II, and 100\%) and in xylene I and II for 3 min, respectively. Finally, these slides were sealed with neutral gum. The adjacent normal breast tissue in each TMA block served as the internal control on the slides. Immunohistochemical staining for PD-L1 was performed on all TMAs.

\section{Assessment Criteria}

The expression of PD-L1 in pretreatment biopsies was assayed with an immunohistochemical method and evaluated under a high-resolution optical microscope to observe the staining of cells. PD-L1 staining was scored in both the tumor cells (TCs) and the PTLCs [16]. PTLCs and TCs staining was identified as positive when clear membranous staining was present in $\geq 1 \%$ of cells [ 17 , 18]. Analysis was conducted by 1 intermediate and 1 senior pathologist, disagreement was resolved by discussion and consensus and, where required, through discussion with a pathologist with content expertise.

Breast cancer intrinsic molecular subtypes were determined by ER, PR, Ki-67, and HER2 status following the 2011 St. Gallen International Expert Consensus recommendations [19]. The pathological response to NACT was assessed by the MP grading system 
Fig. 1. Representative photographs of $\mathrm{PD}$ L1 expression in pretreatment biopsies (all images $\times 400$ magnification). a Positive PD-L1 staining on tumor cells. b Negative PD-L1 staining on tumor cells. c Positive PD-L1 staining on peritumoral lymphocytes. d Negative PD-L1 staining on peritumoral lymphocytes.
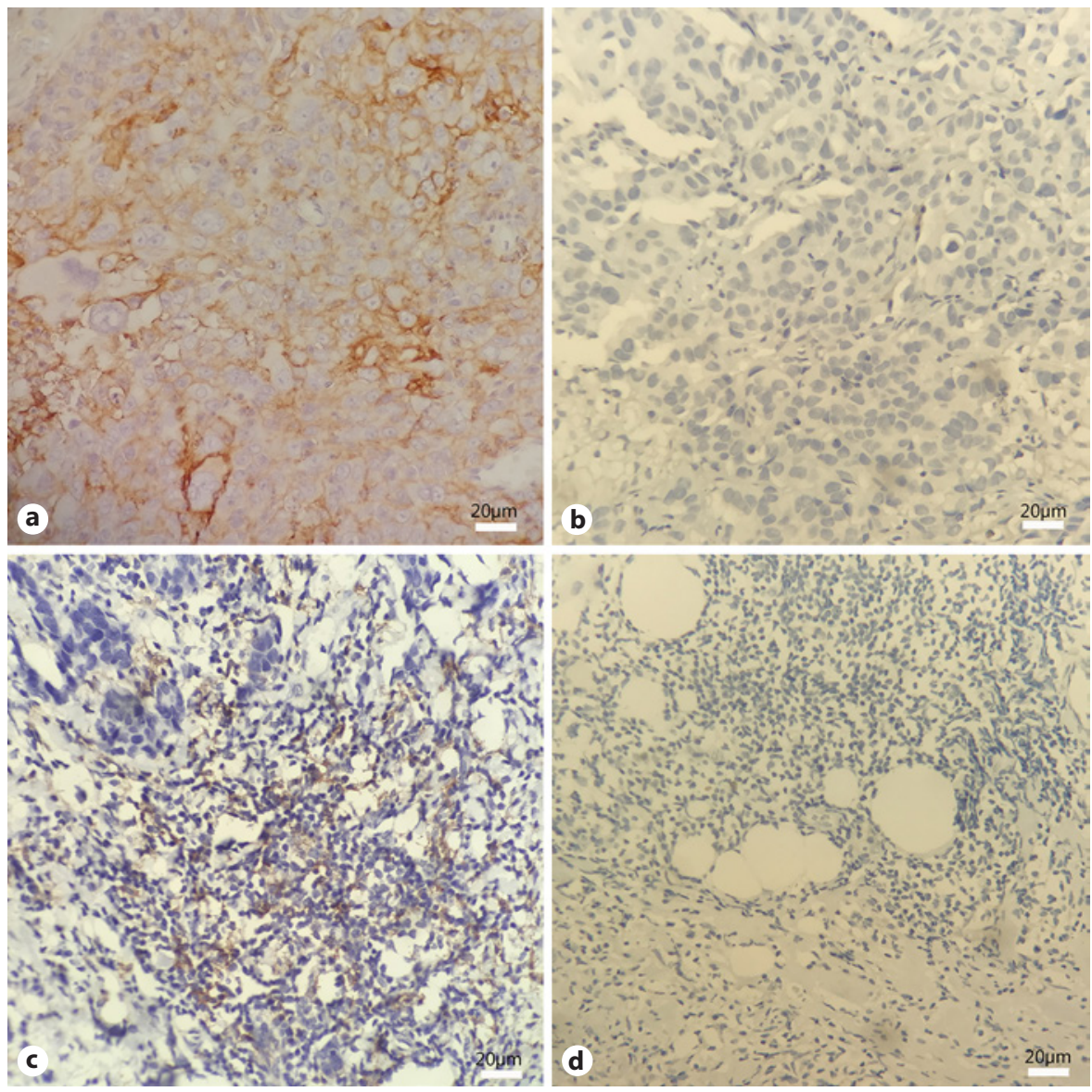

[9], and pCR was defined as MP grade 5. TNM staging was assessed according to the 8th edition of the American Joint Committee on Cancer (AJCC) staging system. The clinical staging of the breast tumors was performed by breast ultrasound, mammography, or MRI. The node status was determined by ultrasound or needle biopsy.

\section{Statistical Analysis}

All data were analyzed using SPSS statistical software (version 22.0; IBM, Armonk, NY, USA). The $\chi^{2}$ and Fisher's exact probability tests were used to analyze differences between qualitative data. The Spearman test was used to analyze correlations between TCs PD-L1 expression and PTLCs PD-L1 expression. Univariate and multivariate logistic regression were used to analyze the associations between PD-L1 expression and MP grades. Statistical results were considered significant with a $p$ value $<0.05$.

\section{Results}

\section{MP Grades and the Response to NACT}

The pathological responses to NACT of 53 patients were assessed by the MP grading system as follows: 10 (18.9\%) patients were appraised as having a grade 5 (G5) response (pCR), 7 (13.2\%) patients as having a grade 4 (G4) response, $10(18.9 \%)$ patients as having a grade 3 (G3) response, $19(35.8 \%)$ patients as having a grade 2
Table 2. PD-L1 expression before NACT of 53 patients with breast cancer

\begin{tabular}{lccll}
\hline & TCs PD-L1 & & $\chi^{2}$ & $r$ \\
& negative & positive & $p$ value & $p$ value \\
\hline PTLCs PD-L1 & & & & \\
$\quad$ Negative & $41(77.4)$ & $4(7.5)$ & 2.68 & 0.30 \\
Positive & $5(9.4)$ & $3(5.7)$ & 0.10 & 0.03 \\
\hline
\end{tabular}

Values are $n$ (\%). TCs PD-L1, PD-L1 expression in tumor cells; PTLCs PD-L1, PD-L1 expression in peritumoral lymphocytes.

(G2) response, and 7 (13.2\%) patients as having a grade 1 (G1) response.

\section{PD-L1 Protein Expression}

Of 53 patients, 12 (22.6\%) patients showed PD-L1 expression, including 4 (7.5\%) patients with staining in TCs only, 5 (9.4\%) patients with staining in PTLCs only, and $3(5.7 \%)$ patients with staining in both TCs and PTLCs. There was no significant difference in PD-L1 expression in pretreatment biopsies between the TCs and PTLCs ( $p>0.05)$, but there was a positive correlation $(r=0.30$, $p=0.03$; Fig. 1; Table 2). 
Table 3. Baseline parameters and distribution of Miller/Payne grades and univariate logistic analysis of predictive factors for Miller/Payne grades in breast cancer patients treated with NACT

\begin{tabular}{|c|c|c|c|c|c|c|c|}
\hline & \multicolumn{2}{|c|}{ Miller/Payne grades, $n(\%)$} & \multirow{2}{*}{$\chi^{2}$} & \multirow[t]{2}{*}{$p$ value } & \multicolumn{3}{|c|}{ Univariate logistic analysis } \\
\hline & $\mathrm{N}$ pCR/G1-4 & $\mathrm{pCR} / \mathrm{G} 5$ & & & OR & $95 \% \mathrm{CI}$ & $p$ value \\
\hline Age & & & 0.22 & 0.64 & & & \\
\hline$<50$ years & $18(78.3)$ & $5(21.7)$ & & & 1 & & \\
\hline$\geq 50$ years & $25(83.3)$ & $5(16.7)$ & & & 0.72 & $0.18-2.86$ & 0.64 \\
\hline Menstrual status & & & 0.02 & 0.88 & & & \\
\hline Premenopausal & $21(84.0)$ & $4(16.0)$ & & & 1 & & \\
\hline Postmenopausal & $22(78.6)$ & $6(21.4)$ & & & 1.43 & $0.35-5.80$ & 0.62 \\
\hline Tumor stage & & & 0.00 & 1.00 & & & \\
\hline $\mathrm{cT} 1-2$ & $35(81.4)$ & $8(18.6)$ & & & 1 & & \\
\hline cT3-4 & $8(80.0)$ & $2(20.0)$ & & & 1.09 & $0.19-6.17$ & 0.92 \\
\hline Nodal status & & & 0.04 & 0.85 & & & \\
\hline $\mathrm{cN}-$ & $5(71.4)$ & $2(28.6)$ & & & 1 & & \\
\hline $\mathrm{cN}+$ & $38(82.6)$ & $8(17.4)$ & & & 0.53 & $0.09-3.21$ & 0.48 \\
\hline ER & & & 1.57 & 0.21 & & & \\
\hline Negative & $18(72.0)$ & $7(28.0)$ & & & 1 & & \\
\hline Positive & $25(89.3)$ & $3(10.7)$ & & & 0.31 & $0.07-1.36$ & 0.12 \\
\hline $\mathrm{PR}$ & & & 0.47 & 0.49 & & & \\
\hline Negative & $18(75.0)$ & $6(25.0)$ & & & 1 & & \\
\hline Positive & $25(86.2)$ & $4(13.8)$ & & & 0.48 & $0.12-1.95$ & 0.31 \\
\hline HER2 & & & 1.82 & 0.18 & & & \\
\hline Negative & $31(86.1)$ & $5(13.9)$ & & & 1 & & \\
\hline Positive & $12(70.6)$ & $5(29.4)$ & & & 2.58 & $0.63-10.55$ & 0.19 \\
\hline $\mathrm{Ki}-67$ & & & 0.00 & 1.00 & & & \\
\hline$<14 \%$ & $4(80.0)$ & $1(20.0)$ & & & 1 & & \\
\hline$\geq 14 \%$ & $39(81.2)$ & $9(18.8)$ & & & 0.92 & $0.09-9.28$ & 0.95 \\
\hline Subtypes & & & 0.62 & 0.88 & & & 0.87 \\
\hline Triple negative & $6(75.0)$ & $2(25.0)$ & & & 1 & & \\
\hline Luminal & $29(82.9)$ & $6(17.1)$ & & & 0.62 & $0.10-3.85$ & 0.61 \\
\hline HER2 & $8(80.0)$ & $2(20.0)$ & & & 0.75 & $0.08-6.96$ & 0.80 \\
\hline TCs PD-L1 & & & 10.87 & 0.00 & & & \\
\hline Negative & $41(89.1)$ & $5(10.9)$ & & & 1 & & \\
\hline Positive & $2(28.6)$ & $5(71.4)$ & & & 20.50 & $3.11-134.94$ & 0.00 \\
\hline PTLCs PD-L1 & & & 3.81 & 0.05 & & & \\
\hline Negative & $39(86.7)$ & $6(13.3)$ & & & 1 & & \\
\hline Positive & $4(50.0)$ & $4(50.0)$ & & & 6.50 & $1.27-33.20$ & 0.02 \\
\hline
\end{tabular}

TCs PD-L1, PD-L1 expression in tumor cells before NACT; PTLCs PD-L1, PD-L1 expression in peritumoral lymphocytes before NACT.

Baseline Parameters and Distribution of MP Grades

There was a significant difference in TCs PD-L1 expression before NACT between the G5 and G1-4 groups $(p=0.00)$ and a marginal difference in PTLCs PD-L1 expression $(p=0.05)$. There was no significant difference in age, menstrual status, clinical tumor stage, nodal status, ER, PR, HER2, Ki-67, and molecular subtypes between the G5 and G1-4 groups ( $p>0.05$; Table 3$)$.

\section{Univariate Analysis of MP Grades}

Univariate logistic regression analysis revealed that G5 response to NACT in breast cancer was significantly associated with TCs or PTLCs PD-L1 positivity (TCs PDL1: $p=0.00$, OR 20.50, 95\% CI 3.11-134.94; PTLCs PD-
L1: $p=0.02$, OR 6.50, 95\% CI 1.27-33.20), while it was unrelated to age, menstrual status, clinical tumor stage, nodal status, ER, PR, HER2, Ki-67, and molecular subtypes (Table 3).

\section{Multivariate Logistic Analysis of MP Grades}

The factors brought into multivariate logistic regression analyzes included age, clinical tumor stage, nodal status, molecular subtypes (luminal, HER2, or triple negative), and PD-L1 expression before NACT (TCs or PTLCs PD-L1 expression was analyzed, respectively). The results showed that G5 response to NACT in breast cancer was significantly associated with TCs or PTLCs PD-L1-positive expression (TCs PD-L1: $p=0.00$, OR 
Table 4. Multivariate logistic analysis of predictive factors for Miller/Payne grades in breast cancer patients treated with NACT

\begin{tabular}{|c|c|c|c|c|c|c|c|}
\hline \multirow[t]{2}{*}{ Variable } & \multirow[t]{2}{*}{$n$} & \multicolumn{3}{|c|}{ TCs PD-L1 } & \multicolumn{3}{|c|}{ PTLCs PD-L1 } \\
\hline & & $\overline{\mathrm{OR}}$ & $95 \% \mathrm{CI}$ & $p$ value & $\overline{\mathrm{OR}}$ & $95 \% \mathrm{CI}$ & $p$ value \\
\hline \multicolumn{8}{|l|}{ Age } \\
\hline$<50$ years & 23 & 1 & & & 1 & & \\
\hline$\geq 50$ years & 30 & 0.74 & $0.13-4.04$ & 0.72 & 0.46 & $0.09-2.35$ & 0.35 \\
\hline \multicolumn{8}{|l|}{ Tumor stage } \\
\hline cT1-2 & 43 & 1 & & & 1 & & \\
\hline cT3-4 & 10 & 1.62 & $0.19-13.94$ & 0.66 & 1.30 & $0.17-9.91$ & 0.80 \\
\hline \multicolumn{8}{|l|}{ Nodal status } \\
\hline $\mathrm{cN}-$ & 7 & 1 & & & 1 & & \\
\hline $\mathrm{cN}+$ & 46 & 2.40 & $0.13-44.68$ & 0.56 & 0.36 & $0.05-2.80$ & 0.36 \\
\hline Subtypes & & & & 0.62 & & & 0.92 \\
\hline Triple negative & 8 & 1 & & & 1 & & \\
\hline Luminal & 35 & 1.03 & $0.08-12.80$ & 0.98 & 1.02 & $0.11-9.42$ & 0.99 \\
\hline HER2 & 10 & 2.81 & $0.14-55.61$ & 0.50 & 1.53 & $0.09-25.17$ & 0.77 \\
\hline \multicolumn{8}{|l|}{ TCs PD-L1 } \\
\hline Negative & 46 & 1 & & & & & \\
\hline Positive & 7 & 42.23 & $3.36-530.90$ & 0.00 & & & \\
\hline \multicolumn{8}{|l|}{ PTLCs PD-L1 } \\
\hline Negative & 45 & & & & 1 & & \\
\hline Positive & 8 & & & & 9.07 & $1.37-60.02$ & 0.02 \\
\hline
\end{tabular}

TCs PD-L1, PD-L1 expression in tumor cells before NACT; PTLCs PD-L1, PD-L1 expression in peritumoral lymphocytes before NACT.

Table 5. Multivariate logistic analysis of predictive factors for Miller/Payne grades in luminal subgroup of breast cancer patients treated with NACT $(n=35)$

\begin{tabular}{|c|c|c|c|c|c|c|c|}
\hline \multirow[t]{2}{*}{ Variable } & \multirow[t]{2}{*}{$n$} & \multicolumn{3}{|c|}{ TCs PD-L1 } & \multicolumn{3}{|c|}{ PTLCs PD-L1 } \\
\hline & & OR & $95 \% \mathrm{CI}$ & $p$ value & OR & $95 \%$ CI & $p$ value \\
\hline \multicolumn{8}{|l|}{ Age } \\
\hline$<50$ years & 16 & 1 & & & 1 & & \\
\hline$\geq 50$ years & 19 & 0.95 & $0.10-8.69$ & 0.96 & 0.39 & $0.03-4.71$ & 0.46 \\
\hline \multicolumn{8}{|l|}{ Tumor stage } \\
\hline cT $1-2$ & 29 & 1 & & & 1 & & \\
\hline cT3-4 & 6 & 1.92 & $0.14-26.74$ & 0.63 & 1.89 & $0.13-27.36$ & 0.64 \\
\hline \multicolumn{8}{|l|}{ Nodal status } \\
\hline $\mathrm{cN}-$ & 6 & 1 & & & 1 & & \\
\hline $\mathrm{cN}+$ & 29 & 3.06 & $0.11-86.90$ & 0.56 & 1.08 & $0.04-27.50$ & 0.97 \\
\hline \multicolumn{8}{|l|}{ TCs PD-L1 } \\
\hline Negative & 30 & 1 & & & & & \\
\hline Positive & 5 & 23.43 & $1.66-331.58$ & 0.02 & & & \\
\hline \multicolumn{8}{|l|}{ PTLCs PD-L1 } \\
\hline Negative & 31 & & & & 1 & & \\
\hline Positive & 4 & & & & 47.89 & $2.47-927.41$ & 0.01 \\
\hline
\end{tabular}

TCs PD-L1, PD-L1 expression in tumor cells before NACT; PTLCs PD-L1, PD-L1 expression in peritumoral lymphocytes before NACT.

42.23, 95\% CI 3.36-530.90; PTLCs PD-L1: $p=0.02$, OR $9.07,95 \%$ CI 1.37-60.02). The other factors were not related to G5 response, including age, clinical tumor stage, nodal status, and molecular subtypes (Table 4).
The same result was found in the luminal subgroup analysis (TCs PD-L1: $p=0.02$, OR 23.43, 95\% CI 1.66331.58; PTLCs PD-L1: $p=0.01$, OR 47.89, 95\% CI $2.47-$ 927.41; Table 5). 


\section{Discussion}

PD-L1 is expressed by a wide range of cancer cell types including breast cancer. PD-L1 interacts with the immune cell surface protein PD-1 to convey an inhibitory signal to TILs to reduce cytotoxic T-cell activity and stimulate regulatory T-cell development, thereby extinguishing the immune response, impeding immune responses, and achieving immune evasion or immune resistance to endogenous antitumor activity $[10,15]$. Recent reports have found that the presence of TILs, including $\mathrm{T}$ cells and dendritic cells, predicts the response to NACT [2023]; PD-L1 expression in breast cancer correlated with TILs [24]. Reports have shown that patients who achieve pCR from neoadjuvant therapy tend to have improved DFS and OS $[4,6-8]$. As patients with PD-L1 positivity would benefit from NACT in breast cancer, we researched on it preliminarily; it is very important to effectively predict response to NACT.

\section{PD-L1 Expression in TCs and MP Grades in Breast Cancer}

In a total of 245 primary and 40 metastatic breast carcinomas evaluated with TMAs using IHC, the positive rates of TCs PD-L1 staining were 12\% [25], which is consistent with the $13.2 \%$ found in the present study. Wimberly et al. [12] found that the immunofluorescence expression of PD-L1 in 80 breast cancer patients treated with NACT was associated with pCR and was nearing significance in predicting $\mathrm{pCR}$ by multivariate analysis. Using DNA microarray technology, Sabatier et al. [26] found that a high expression of PD-L1 mRNA was associated with pCR in all 265 breast cancer patients treated with NACT by univariate analysis, whereas none of the tested clinicopathological features (including age, ER or PR or HER2 status, pathological type) was. There was a predictive value of PD-L1 expression in basal and HER2-enriched cases, but not in luminal A, luminal B, or normal-like cases. In our study, G5 response to NACT in breast cancer was significantly associated with TCs PD-L1 positivity in pretreatment biopsies both in the total breast cancer population and in luminal subtype. Similar to other studies that used IHC technology, PD-L1 protein expression in TCs was found to be a predictive biomarker of pCR both in hormone receptor-positive patients and all 94 locally advanced breast cancer patients treated with NACT(with paclitaxel plus cisplatin weekly regimens, which was not the preferred therapy) by both univariate and multivariate logistic regression analysis [27]. However, the predictive value of PTLCs PD-L1 expression for $\mathrm{pCR}$ was not analyzed, and the molecular subtypes and nodal status were not included in the analysis, which were included in our study.
PD-L1 Expression in PTLCs and MP Grades in Breast Cancer

Recent reports have found that the presence of TILs, including $\mathrm{T}$ cells and dendritic cells, predicts the response to NACT [20-22]; PD-L1 expression in breast cancer correlated with TILs [24] and predicted pCR to NACT $[28,29]$. Our research confirmed that there was a positive correlation between TCs PD-L1 expression and PTLCs PD-L1 expression before NACT in breast cancer $(r=0.30, p=0.03$; Table 2), while G5 response to NACT in breast cancer was significantly associated with TCs or PTLCs PD-L1-positive expression before NACT both in the total breast cancer population and in luminal subtype.

\section{Predictive Biomarkers for MP Grade 5 ( $p C R$ ) in Breast Cancer}

NACT is the preferred treatment for patients with locally advanced breast cancer. According to previous studies on breast cancer NACT, the clinical response rates ranged from 50 to $90 \%$, and the $\mathrm{pCR}$ rates were between 4 and $60 \%$. The pCR rate is associated with time from start of NACT to surgery, NACT cycles, ER or PR or HER2 status, breast cancer subtype, Nottingham grade, mitotic count, Ki-67 score, TILs, stromal infiltration lymphocytes, and/or chemotherapy strategy (specially HER2-directed therapy with trastuzumab). In addition, the definition of pCR (applied to axilla and breast, or to breast only), breast cancer subtype (e.g., HER2+ type regardless of ER or PR status, luminal subtype was defined as ER+ and/or PR+ and HER2-), or other variables included varied in different reports, so that results also varied by univariate or multivariate analysis; sometimes, because variables are strongly associated, predictive factors cannot both be included in a significant multivariate model $[2,6,9,23,30-33]$. Generally, tumor subtype was associated with pCR rate; the HER2 (regardless of ER or PR status) and triple-negative subtypes had higher $\mathrm{PCR}$ rates than the luminal subtype (ER+ or PR+ and HER2-) [23, 33]. An independent association between pCR and breast cancer subtype was found by multivariate analysis in a meta-analysis [33], while another report showed that clinical stage, HER2 positivity, and mitotic count were significant predictors of pCR in multivariate analysis [23]. In our study, the pCR (G5) rate was $18.9 \%$, applying subtypes recommended by the 2011 St. Gallen International Expert Consensus; G5 response to NACT in breast cancer was significantly associated with TCs or PTLCs PD-L1 positivity before NACT both in the total breast cancer population and in luminal subtype by multivariate logistic regression. So, more rigorous studies to predict MP G5 or pCR need to be designed. 


\section{Conclusion}

G5 response to NACT in breast cancer was significantly associated with TCs or PTLCs PD-L1-positive expression before NACT, and PD-L1 promises to be a potential predictive biomarker for response to NACT in breast cancer.

\section{Acknowledgement}

We thank all the teachers of the Pathology Department at Weifang People's Hospital for their help and support.

\section{Statement of Ethics}

The patients gave their written informed consent, and the study protocol was approved by the ethics committee of Weifang People's Hospital.

\section{Conflict of Interest Statement}

The authors have no conflicts of interest to declare.

\section{Funding Sources}

The Funding Project ZR2009CM081 was supported by the Shandong Provincial Natural Science Foundation.

\section{Author Contributions}

All authors contributed to the study conception and design. Guarantors of the integrity of the entire study were F.-R.H., Y.-X.Z., and R.-Z.M. Study concept and design were performed by F.-R.H. Literature research was performed by C.L., F.-R.H., R.-Z.M., and Y.-H.G. Clinical studies were performed by C.L., F.-R.H., Y.-H.G., Y.-N.Z., Y.-T.Z., F.-M.C., and S.-G.Z. Experimental studies and data analysis were performed by C.L., Y.-X.Z., G.-Y.H., H.W., and Y.-P.Z. Statistical analysis was performed by C.L., Y.-H.G., M.C.W., and F.-R.H. Manuscript preparation was performed by C.L. Manuscript editing was performed by C.L. and F.-R.H.

\section{References}

1 Bray F, Ferlay J, Soerjomataram I, Siegel RL, Torre LA, Jemal A. Global cancer statistics 2018 GLOBOCAN estimates of incidence and mortality worldwide for 36 cancers in 185 countries. CA Cancer J Clin. 2018 Nov;68(6):394-424.

2 Killelea BK, Yang VQ, Mougalian S, Horowitz NR, Pusztai L, Chagpar AB, et al. Neoadjuvant chemotherapy for breast cancer increases the rate of breast conservation: results from the National Cancer Database. J Am Coll Surg. 2015 Jun;220(6):1063-9.

3 Puig CA, Hoskin TL, Day CN, Habermann EB, Boughey JC. National Trends in the Use of Neoadjuvant Chemotherapy for Hormone Receptor-Negative Breast Cancer: A National Cancer Data Base Study. Ann Surg Oncol. 2017 May;24(5):1242-50.

4 Cortazar P, Zhang L, Untch M, Mehta K, Costantino JP, Wolmark N, et al. Pathological complete response and long-term clinical benefit in breast cancer: the CTNeoBC pooled analysis. Lancet. 2014 Jul;384(9938):164-72.

5 Qi CZ, Huang M, Haiderali A, Xie J, Zhou ZY, $\mathrm{Wu}$ EQ, et al. Association between Pathological Complete Responses and Long-Term Survival Outcomes among Triple-Negative Breast Cancer Patients Receiving Neoadjuvant Chemotherapy. J Natl Compr Canc Netw. 2019;17(3.5):HSR19-106.

6 LeVasseur N, Sun J, Gondara L, Diocee R, Speers C, Lohrisch C, et al. Impact of pathologic complete response on survival after neoadjuvant chemotherapy in early-stage breast cancer: a population-based analysis. J Cancer Res Clin Oncol. 2020 Feb;146(2):529-36.

7 von Minckwitz G, Untch M, Blohmer JU, Costa SD, Eidtmann H, Fasching PA, et al. Definition and impact of pathologic complete response on prognosis after neoadjuvant chemotherapy in various intrinsic breast cancer subtypes. J Clin Oncol. 2012 May;30(15): 1796-804.
8 von Minckwitz G, Fontanella C. Selecting the neoadjuvant treatment by molecular subtype: how to maximize the benefit? Breast. 2013 Aug;22 Suppl 2:S149-51.

9 Ogston KN, Miller ID, Payne S, Hutcheon AW, Sarkar TK, Smith I, et al. A new histological grading system to assess response of breast cancers to primary chemotherapy: prognostic significance and survival. Breast. 2003 Oct;12(5):320-7.

10 Taube JM, Anders RA, Young GD, Xu H, Sharma R, McMiller TL, et al. Colocalization of Inflammatory Response with B7-H1 Expression in Human Melanocytic Lesions Supports an Adaptive Resistance Mechanism of Immune Escape. Sci Transl Med. 2012 Mar; 4(127):127ra37.

11 Beckers RK, Selinger CI, Vilain R, Madore J, Wilmott JS, Harvey K, et al. PDL1 expression in triple-negative breast cancer is associated with tumour-infiltrating lymphocytes and improved outcome. Histopathology. 2016; 48(1):S146-7.

12 Wimberly H, Brown JR, Schalper K, Haack H, Silver MR, Nixon C, et al. PD-L1 Expression Correlates with Tumor-Infiltrating Lymphocytes and Response to Neoadjuvant Chemotherapy in Breast Cancer. Cancer Immunol Res. 2015 Apr;3(4):326-32.

13 Gatalica Z, Snyder C, Maney T, Ghazalpour A, Holterman DA, Xiao N, et al. Programmed cell death 1 (PD-1) and its ligand (PD-L1) in common cancers and their correlation with molecular cancer type. Cancer Epidemiol Biomarkers Prev. 2014 Dec; 23(12):2965-70

14 Cimino-Mathews A, Thompson E, Taube JM, Ye X, Lu Y, Meeker A, et al. PD-L1 (B7-H1) expression and the immune tumor microenvironment in primary and metastatic breast carcinomas. Hum Pathol. 2016 Jan;47(1):5263.
15 Nguyen LT, Ohashi PS. Clinical blockade of PD1 and LAG3 - potential mechanisms of action. Nat Rev Immunol. 2015 Jan;15(1):4556.

16 Herbst RS, Soria JC, Kowanetz M, Fine GD, Hamid O, Gordon MS, et al. Predictive correlates of response to the anti-PD-L1 antibody MPDL3280A in cancer patients. Nature. 2014 Nov;515(7528):563-7.

17 Pang C, Yin L, Zhou X, Lei C, Tong R, Huang $\mathrm{M}$, et al. Assessment of programmed cell death ligand-1 expression with multiple immunohistochemistry antibody clones in nonsmall cell lung cancer. J Thorac Dis. 2018 Feb; 10(2):816-24.

18 Buisseret L, Garaud S, de Wind A, Van den Eynden G, Boisson A, Solinas C, et al. Tumorinfiltrating lymphocyte composition, organization and PD-1/ PD-L1 expression are linked in breast cancer. OncoImmunology. 2016 Dec;6(1):e1257452.

19 Goldhirsch A, Wood WC, Coates AS, Gelber RD, Thürlimann B, Senn HJ; Panel members. Strategies for subtypes-dealing with the diversity of breast cancer: highlights of the St. Gallen International Expert Consensus on the Primary Therapy of Early Breast Cancer 2011. Ann Oncol. 2011 Aug;22(8): 1736-47.

20 Asano Y, Kashiwagi S, Goto W, Takada K, Takahashi K, Hatano T, et al. Prediction of Treatment Response to Neoadjuvant Chemotherapy in Breast Cancer by Subtype Using Tumor-infiltrating Lymphocytes. Anticancer Res. 2018 Apr;38(4):2311-21.

21 Denkert C, von Minckwitz G, Darb-Esfahani S, Lederer B, Heppner BI, Weber KE, et al. Tumour-infiltrating lymphocytes and prognosis in different subtypes of breast cancer: a pooled analysis of 3771 patients treated with neoadjuvant therapy. Lancet Oncol. 2018 Jan; 19(1):40-50. 
22 Hamy AS, Bonsang-Kitzis H, De Croze D, Laas E, Darrigues L, Topciu L, et al. Interaction between Molecular Subtypes and Stromal Immune Infiltration before and after Treatment in Breast Cancer Patients Treated with Neoadjuvant Chemotherapy. Clin Cancer Res. 2019 Nov;25(22):6731-41.

23 Li XB, Krishnamurti U, Bhattarai S, Klimov S, Reid MD, O’Regan R, et al. Biomarkers Predicting Pathologic Complete Response to Neoadjuvant Chemotherapy in Breast Cancer. Am J Clin Pathol. 2016 Jun;145(6):871-8.

24 Noske A, Möbus V, Weber K, Schmatloch S, Weichert W, Köhne $\mathrm{CH}$, et al. Relevance of tumour-infiltrating lymphocytes, PD-1 and PD-L1 in patients with high-risk, nodal-metastasised breast cancer of the German Adjuvant Intergroup Node-positive study. Eur J Cancer. 2019 Jun;114:76-88.

25 Dill EA, Gru AA, Atkins KA, Friedman LA, Moore ME, Bullock TN, et al. PD-L1 Expression and Intratumoral Heterogeneity Across Breast Cancer Subtypes and Stages: An Assessment of 245 Primary and 40 Metastatic Tumors. Am J Surg Pathol. 2017 Mar;41(3): 334-42.
26 Sabatier R, Finetti P, Mamessier E, Adelaide J, Chaffanet M, Ali HR, et al. Prognostic and predictive value of PDL1 expression in breast cancer. Oncotarget. 2015 Mar;6(7):5449-64.

27 Wu Z, Zhang L, Peng J, Xu S, Zhou L, Lin Y, et al. Predictive and prognostic value of PDL1 protein expression in breast cancer patients in neoadjuvant setting. Cancer Biol Ther. 2019; 20(6):941-7.

28 Kitano A, Ono M, Yoshida M, Noguchi E, Shimomura A, Shimoi T, et al. Tumour-infiltrating lymphocytes are correlated with higher expression levels of PD-1 and PD-L1 in early breast cancer. ESMO Open. 2017 May; 2(2):e000150.

29 Van Berckelaer C, Rypens C, van Dam P, Pouillon L, Parizel M, Schats KA, et al. Infiltrating stromal immune cells in inflammatory breast cancer are associated with an improved outcome and increased PD-L1 expression. Breast Cancer Res. 2019 Feb;21(1):28.
30 Bear HD, Anderson S, Smith RE, Geyer CE Jr, Mamounas EP, Fisher B, et al. Sequential preoperative or postoperative docetaxel added to preoperative doxorubicin plus cyclophosphamide for operable breast cancer: National Surgical Adjuvant Breast and Bowel Project Protocol B-27. J Clin Oncol. 2006 May;24(13):2019-27.

31 van der Hage JA, van de Velde CJ, Julien JP Tubiana-Hulin M, Vandervelden C, Duchateau L. Preoperative chemotherapy in primary operable breast cancer: results from the European Organization for Research and Treatment of Cancer trial 10902. J Clin Oncol. 2001 Nov;19(22):4224-37.

32 Rastogi P, Anderson SJ, Bear HD, Geyer CE, Kahlenberg MS, Robidoux A, et al. Preoperative chemotherapy: updates of National Surgical Adjuvant Breast and Bowel Project Protocols B-18 and B-27. J Clin Oncol. 2008 Feb; 26(5):778-85.

33 Houssami N, Macaskill P, von Minckwitz G, Marinovich ML, Mamounas E. Meta-analysis of the association of breast cancer subtype and pathologic complete response to neoadjuvant chemotherapy. Eur J Cancer. 2012 Dec; 48(18):3342-54. 